\title{
ON A THEOREM OF PALEY
}

\author{
BY LEOPOLD FEJÉR
}

1. Introduction. In $1910 \mathrm{I}$ published the following theorem. $\dagger$

THEOREM 1. Let $f(x)$ be integrable in the interval $0 \leqq x \leqq 2 \pi$ and such that

$$
|f(x)| \leqq M, \quad(0 \leqq x \leqq 2 \pi),
$$

and let the Fourier coefficients $a_{n}, b_{n}$ of $f(x)$ satisfy the conditions

$$
\left|a_{n}\right| \leqq \frac{A}{n}, \quad\left|b_{n}\right| \leqq \frac{B}{n}, \quad(n=1,2, \cdots),
$$

where $M, A, B$ are non-negative constants. Then, if $s_{n}(x)$ denotes the sum of the $(n+1)$ first terms of the Fourier series of $f(x)$, the following inequality holds:

(3) $\left|s_{n}(x)\right| \leqq M+A+B, \quad(0 \leqq x \leqq 2 \pi, n=0,1,2, \cdots)$.

This criterion of boundedness of the partial sums of the Fourier series of a bounded function follows immediately from the elementary fact that, when $|f(x)| \leqq M$ in $(0,2 \pi)$, then also $\left|S_{n}(x)\right| \leqq M$ in $(0,2 \pi),(n=0,1,2, \cdots)$, where $S_{n}(x)$ is the arithmetic mean of the first $(n+1)$ partial sums of the Fourier series of $f(x)$. Indeed, for an arbitrary infinite series

$$
u_{0}+u_{1}+u_{2}+\cdots+u_{n}+\cdots \text {, }
$$

there exists the relation

$$
s_{n}=S_{n}+(n+1)^{-1} \sum_{\nu=1}^{n} \nu u_{\nu},
$$

from which Theorem 1 follows at once.

2. Paley's Theorem. Leaving aside various interesting considerations related to Theorem 1 which are known, I shall now pass on to a second boundedness criterion for the partial sums

$\dagger$ Sur les sommes partielles de la série de Fourier, Comptes Rendus, vol. 150 (1910), pp. 1299-1302. 
of the Fourier series of a bounded function, which was published recently by Paley. $\dagger$

TheORem 2 (Paley). If the integrable function $f(x)$ satisfies the condition (1), while all its Fourier coefficients are non-negative,

$$
a_{n} \geqq 0, \quad b_{n} \geqq 0,
$$

then $\ddagger$

$$
\left|s_{n}(x)\right| \leqq 10 M, \quad(0 \leqq x \leqq 2 \pi, n=0,1,2, \cdots) .
$$

The very short and simple proof of Theorem 2 given by Paley is based, on the one hand, again on the inequality $\left|S_{n}(x)\right| \leqq M$, and, on the other hand, on the theorem of S. Bernstein, according to which the absolute value of the derivative of a trigonometric polynomial of order $n$ does not exceed $n$ times the maximum of the absolute value of the polynomial.

In a personal conversation with Paley at the International Congress of Mathematicians in Zurich, in September 1932, I stated that the proof of his Theorem 2, as well as that of my Theorem 1, may be derived from the inequality $\left|S_{n}(x)\right| \leqq M$ alone, if one uses an elementary device, which I have used for other purposes. $\$$ My proof, which I communicated to Paley in a letter in September, 1932, runs as follows.

3. Proof of Theorem 2. Let $f(x)$ (integrable and periodic, of period $2 \pi$ ) generate the Fourier series

$$
f(x) \sim a_{0}+\sum_{\nu=1}^{\infty}\left(a_{\nu} \cos \nu x+b_{\nu} \sin \nu x\right) .
$$

Let also

$$
|f(x)| \leqq M, \quad(0 \leqq x \leqq 2 \pi) .
$$

For the arithmetic mean $S_{n}(x)$ of the first $(n+1)$ partial sums of the series (8) we have the inequality

$\dagger$ On Fourier series with positive coefficients, Journal of the London Mathematical Society, vol. 7 (1932), pp. 205-208.

$\ddagger$ In $\$ 3$ of this note I show that this inequality holds with the coefficient 4 instead of 10 , while the coefficient 1 would be too small.

§ See my note, Über einen S. Bernsteinschen Satz, und über die Szegösche Verschärfung desselben, Bulletin of the Calcutta Mathematical Society, vol. 20 (1930), pp. 49-54. 


$$
\left|S_{n}(x)\right| \leqq M, \quad(0 \leqq x \leqq 2 \pi, n=0,1,2, \cdots),
$$

and in particular, for $x=0$,

$$
\left|S_{n}(0)\right| \leqq M, \quad(n=0,1,2, \cdots) .
$$

Inequality (11) expresses the fact that the arithmetic means of the series

$$
a_{0}+a_{1}+a_{2}+\cdots+a_{n}+\cdots
$$

are all $\leqq M$ in absolute value. Since, by hypothesis, the terms of this series are non-negative, the series (12) must converge to a sum $\leqq M$. Hence

$$
a_{0}+a_{1}+\cdots+a_{n} \leqq M
$$

and consequently

$$
\left|a_{0}+a_{1} \cos x+\cdots+a_{n} \cos n x\right| \leqq M .
$$

Thus we see that the central point of the proof is to estimate the sum $\sum_{\nu=1}^{n} b_{\nu} \sin \nu x$. Since, by (8),

$$
\phi(x) \equiv \frac{1}{2}[f(x)-f(-x)] \sim \sum_{\nu=1}^{\infty} b_{\nu} \sin \nu x,
$$

while

$$
|\phi(x)| \leqq M
$$

we have only to estimate partial sums of the sine series

$$
\phi(x) \sim \sum_{\nu=1}^{\infty} b_{\nu} \sin \nu x
$$

under the hypotheses that, for each $x,|\phi(x)| \leqq M$, while all $b_{\nu} \geqq 0,(\nu=1,2, \cdots)$.

In view of the general relation (5) and of the fact that all the coefficients $b_{\nu}$ are non-negative, we have only to estimate the sum

$$
b_{1}+2 b_{2}+\cdots+n b_{n} .
$$

I am turning to this now, after having used, so far, the old method of Paley. I am retaining the previous notation $f(x)$ instead of $\phi(x)$. I say first that, if 


$$
f(x) \sim b_{1} \sin x+b_{2} \sin 2 x+\cdots+b_{n} \sin n x+\cdots,
$$

then

$$
\begin{aligned}
2 \sin n x f(x) \sim b_{n} & +\left(b_{n-1}+b_{n+1}\right) \cos x+\cdots \\
& +\left(b_{1}+b_{2 n-1}\right) \cos (n-1) x+b_{2 n} \cos n x \\
& +\left(b_{2 n+1}-b_{1}\right) \cos (n+1) x \\
& +\left(b_{2 n+2}-b_{2}\right) \cos (n+2) x+\cdots .
\end{aligned}
$$

Indeed, if we expand $2 \sin n x f(x)$ in a cosine series, then the constant term is

$$
\alpha_{0}=\frac{1}{\pi} \int_{0}^{\pi} 2 \sin n x f(x) d x=\frac{2}{\pi} \int_{0}^{\pi} f(x) \sin n x d x=b_{n},
$$

while the coefficient of $\cos k x$ is

$$
\begin{aligned}
\alpha_{k} & =\frac{2}{\pi} \int_{0}^{\pi} 2 \sin n x f(x) \cos k x d x \\
& =\frac{2}{\pi} \int_{0}^{\pi} f(x)[\sin (n+k) x+\sin (n-k) x] d x=b_{n+k}+b_{n-k}
\end{aligned}
$$

if we agree to write $b_{0}=0$, and $b_{-l}=-b_{l},(l=1,2,3, \cdots)$. Now apply the inequality $\left|S_{\nu}(x)\right| \leqq M$ to the cosine series (19) at the point $x=0$, and for $\nu=n-1$. Since $|2 \sin n x f(x)| \leqq 2 M$, $(0 \leqq x \leqq 2 \pi)$, we get

$$
\begin{aligned}
\left|S_{n-1}(0)\right|= & \frac{1}{n} \mid n b_{n}+(n-1)\left(b_{n-1}+b_{n+1}\right) \\
& +\cdots+1 \cdot\left(b_{1}+b_{2 n-1}\right) \mid \leqq 2 M,
\end{aligned}
$$

so that

$$
\begin{aligned}
\mid 1 \cdot\left(b_{1}+b_{2 n-1}\right) & +2\left(b_{2}+b_{2 n-2}\right)+\cdots \\
& +(n-1)\left(b_{n-1}+b_{n+1}\right)+n b_{n} \mid \leqq 2 n M .
\end{aligned}
$$

This inequality holds for an arbitrary sine series (18) if only $|f(x)| \leqq M$. When, in addition, all the coefficients $b_{\nu}$ are nonnegative, (20) yields

$$
\begin{aligned}
\left(b_{1}+b_{2 n-1}\right) & +2\left(b_{2}+b_{2 n-2}\right)+\cdots \\
& +(n-1)\left(b_{n-1}+b_{n+1}\right)+n b_{n} \leqq 2 n M
\end{aligned}
$$


and, a fortiori,

$$
b_{1}+2 b_{2}+\cdots+n b_{n} \leqq 2 n M .
$$

Relation (5) gives now

$$
\left|b_{1} \sin x+\cdots+b_{n} \sin n x\right| \leqq M+2 n M /(n+1) \leqq 3 M .
$$

Combining this with (14), we obtain the final result

$$
\left|s_{n}(x)\right| \leqq M+3 M=4 M \text {, }
$$

which is the theorem of Paley (with the coefficient 10 replaced by 4$). \dagger$

4. Remarks. In connection with my conversation with Paley in Zurich I raised the question as to a relationship between the two boundedness criteria, Theorems 1 and 2, to which Paley answered immediately by proving that Theorem 1 follows from Theorem 2. Paley, however, went considerably further. This he did in a letter, of which a part that pertains to the subject is reproduced in $\$ 5$ of the present note. Here I shall make a few explanatory comments. In Theorem 1 , in addition to the inequality $|f(x)| \leqq M$, I make the assumption $-C \leqq n a_{n} \leqq C$, $-C \leqq n b_{n} \leqq C, C \geqq 0$, which is of Hardy's type. These assumptions, as we have seen, imply the uniform boundedness of $s_{n}(x)$. If, in addition, the function $f(x)$ is assumed to be continuous (and periodic of period $2 \pi$ ), then its Fourier series converges uniformly (for it is uniformly $(C 1)$ summable, and hence, by a theorem of Hardy, converges uniformly). Paley has discovered deeper theorems, stating that the conclusions above still hold if the assumption of the "two-sided" boundedness of $n a_{n}, n b_{n}$ (Hardy), made in my Theorem 1 , is replaced by the assumption of the "one-sided" boundedness (Landau), $-C \leqq n a_{n},-C \leqq n b_{n}$. Thus Paley discovered the following theorem.

$\dagger$ Let me indicate, in passing, another application of the inequality (21). If, under the assumptions above, the sequence $\left\{b_{n}\right\}$ is not only non-negative, but also convex (non-concave) from below, then

$$
b_{n} \leqq \frac{1}{2}\left(b_{n-1}+b_{n+1}\right) \leqq \cdots \leqq \frac{1}{2}\left(b_{2}+b_{2 n-2}\right) \leqq \frac{1}{2}\left(b_{1}+b_{2 n-1}\right),
$$

so that, by (21),

$$
1 \cdot 2 b_{n}+2 \cdot 2 b_{n}+\cdots+(n-1) \cdot 2 b_{n}+n b_{n}=n^{2} b_{n} \leqq 2 n M
$$

or, finally, $b_{n} \leqq 2 M / n,(n=1,2,3, \cdots)$. 
If $f(x)$ is integrable and $|f(x)| \leqq M$, and if the Fourier coeffcients of $f(x)$ satisfy the conditions $n a_{n} \geqq-C, n b_{n} \geqq-C$, then the partial sums of the Fourier series of $f(x)$ are uniformly bounded. If, in addition, $f(x)$ is continuous, then the Fourier series of $f(x)$ converges uniformly for all real values of $x$. $\dagger$

This essentially more general theorem, contains my Theorem 1 as well as Paley's Theorem 2 as special cases.

5. Extract from a Letter $\ddagger$ of Paley. "... As we surmised in Zurich, the following theorem is true (indeed trivial):

"Theorem. If $|f| \leqq 1, n a_{n} \geqq-A, n b_{n} \geqq-A$, then the partial sums of the Fourier series are uniformly bounded. [Of course your method applies here again at once.]

"What is not quite so trivial, but still not very difficult is the following theorem.

"Theorem. If $f$ is continuous, $n a_{n} \geqq-A, n b_{n} \geqq-A$, then the Fourier series of $f$ is uniformly convergent.

"It is sufficient to prove the following lemma.

"Lemma. Let $|f| \leqq B$, and $n a_{n} \geqq-A, n b_{n} \geqq-A$; let $\delta$ be an arbitrary positive number. Then

$$
\left|s_{n}(\theta)\right| \leqq A \delta+M_{\delta} B,
$$

where $M_{\partial}$ depends only on $\delta$.

"To prove the lemma, we observe that

$$
s_{n}=\sigma_{n}^{*}+R
$$

where $\sigma_{n}^{*}$ is

$$
\begin{aligned}
1+\cdots+A_{m}(\theta) & +\frac{n-m-1}{n-m} A_{m+1}(\theta) \\
& +\frac{n-m-2}{n-m} A_{m+2}(\theta)+\cdots+\frac{1}{n-m} A_{n-1}(\theta),
\end{aligned}
$$

$\dagger$ Later, but independently of Paley, the same results have been found by O. Szász, Zur Konvergenztheorie der Fourierschen Reihen, Acta Mathematica, vol. 61 (1933), pp. 185-201.

$\ddagger$ Dated October 5, 1932, at the Massachusetts Institute of Technology. 
and $m / n$ lies between constants which depend on $\delta$. Then it is quite easy to prove that

$$
\left|\sigma_{n}^{*}(\theta)\right|=\left|n \sigma_{n}(\theta)-m \sigma_{m}(\theta)\right| /(n-m) \leqq M B,
$$

while your argument shows at once that $\left|R_{n}(\theta)\right| \leqq 2 B+\vartheta A$, where $\vartheta$ is small by choice of $m / n \ldots . " \dagger$

UNIVERSITY OF BUDAPEST

\section{NOTE ON THE FORM OF A FIRST-ORDER PARTIAL DIFFERENTIAL EQUATION}

BY A. B. BROWN

In this paper we give a simple proof of the fact that the non-singular solutions of a first-order partial differential equation can be obtained by equating to zero solutions of an associated equation in which the dependent variable does not appear explicitly. The usual proof $\S$ of this property makes extensive use of the complete integral, and to be given rigorously would require considerations at one stage nearly as involved as our entire proof.|| Our proof has no reference to complete integrals. The results, as usual, hold in the small. Interest in this question arises from the treatments of equations in which the unknown does not appear explicitly.

TheOREM. Let $f\left(x_{1}, \cdots, x_{n}, z, p_{1}, \cdots, p_{n}\right)=f(x, z, p)$ be of class $C^{\prime \prime} \mathbb{T}$ in a neighborhood of an initial element $\left(a, b, p^{0}\right)$ for which $f=0$ and $f_{p_{1}} \neq 0$. Let

$\dagger$ Professor Fekete, to whom I communicated this letter of Paley in September 1933, has worked out completely the proof sketched by Paley. Moreover, Fekete generalized considerably Paley's theorem and extended it also to the trigonometric series of $\mathrm{H}$. Bohr.

$\ddagger$ Presented to the Society, March 31, 1934.

$\S$ See, for example, E. Goursat, Équations aux Dérivées Partielles, 1921, pp. 48-49 and 159 .

\| A complete integral yielding elements at a given point does not necessarily provide any given integral element at the point.

If A function of class $C^{(k)}$ is one having continuous $k$ th partial derivatives. 